\title{
Collateral Predictors of Neovascularization After Indirect Revascularization in Adult Patients with Moyamoya Disease: A Single-Center Retrospective Study
}

Kefang Dai

Capital Medical University

Zhiyong Shi

Nanjing Drum Tower Hospital

Xingju Liu

Capital Medical University

Rong Wang

Capital Medical University

Yan Zhang

Capital Medical University

Dong Zhang ( $\sim$ zhangdong0660@aliyun.com )

Capital Medical University

\section{Research Article}

Keywords: Moyamoya disease, Neovascularization, Risk factors, Clinical outcome

Posted Date: November 3rd, 2021

DOl: https://doi.org/10.21203/rs.3.rs-1004483/v1

License: (c) (i) This work is licensed under a Creative Commons Attribution 4.0 International License.

Read Full License 


\section{Abstract \\ Objective.}

The underlying factors of neovascularization after indirect bypass in the adult patients with moyamoya disease (MMD) remained unknow. The aim of this study was to explore potential predictors based on collateral characteristics for neovascularization after indirect bypass in adult MMD patients.

\section{Methods.}

The adult MMD patients treated by indirect bypass in a single-institution from August 2012 and January 2018 were retrospectively selected into our research. The collaterals based on cerebral angiography were classified into the following subtypes: intracerebral anastomosis, duro-cortical anastomosis, and leptomeningeal anastomosis. Neovascularization evaluation was based on Matsushima classification, with "good" collateral formation in level 2 and 3 , and poor formation in level 0 and 1 . Univariate and multivariate analyses were performed to identify neovascularization predictors after indirect bypass.

\section{Results.}

A total of 86 patients (97 hemispheres) (mean \pm SD age $35.06 \pm 15.09$ years, range 18-61 years) were retrospectively included. Preoperative collateral circulation included intracerebral anastomosis in 49 (50.5\%) cases, duro-cortical anastomosis in 19 (19.6\%) cases, and leptomeningeal anastomosis in 29 (29.9\%) cases, respectively. Postoperative good neovascularization was observed in 56 (57.7\%) hemispheres. Multivariate analysis showed that intracerebral anastomosis $(\mathrm{P}<0.001, \mathrm{OR}[95 \% \mathrm{Cl}] 2.984$ [2.031-5.437]) was associated with favorable neovascularization, whereas older age $(P<0.001,0 R$ [95\% $\mathrm{Cl}] 0.827$ [0.793-0.916]) and hemorrhagic onset $(\mathrm{P}<0.001$, OR [95\% Cl] 0.138 [0.054-0.353]) were significantly associated with poor neovascularization. Hemispheres in the good neovascularization had lower modified Rankin scale score, and better long-term improvement than those in the poor neovascularization.

\section{Conclusions.}

Hemorrhagic onset and old age predict poor neovascularization after indirect bypass, while duro-cortical anastomosis and intracerebral anastomosis predict good neovascularization. Good neovascularization was associated with better long-term outcomes. The current study provides a basis for the selection of surgical procedure for MMD candidates.

\section{Introduction}


Moyamoya disease (MMD) characterized by a progressive stenosis or occlusion of terminal portion of bilateral internal carotid artery (ICA), proximal portion of middle cerebral artery (MCA) and/or proximal anterior cerebral artery (ACA), and formation of moyamoya vessels at the base of brain ${ }^{1-3}$. Cerebral revascularization was recommended as the mainstream of treatment for patients with MMD, including direct bypass, indirect bypass and combined bypass ${ }^{4-7}$. Direct revascularization may be considered as the first-line treatment over indirect methods for adult patients with MMD, while indirect revascularization may be considered as an alternative to direct methods in adult MMD when the latter is not available $e^{6,8-}$ 10. Previous literature reported that indirect bypass was equivalent to direct bypass or combined bypass for the prevention of stroke recurrence in adult patients with MMD. ${ }^{9,11-13}$ Postoperative neovascularization was associated with favorable clinical outcome ${ }^{14}$. However, other underlying factors of neovascularization after indirect bypass in the adult patients with MMD remained unknow. Zhao $Y$ et al. reported abundant moyamoya vessels contributed to postoperative neovascularization ${ }^{15}$. Identifying other potential risk factors of postoperative neovascularization after indirect bypass was beneficial to benefit adult patients with MMD from this procedure. The aim of this study was to explore potential predictors based on collateral characteristics for neovascularization after indirect bypass in adult MMD patients.

\section{Materials And Methods}

\section{Patient Selection}

The research was approved by the Ethics Committee of Beijing Tiantan Hospital and written informed consent was obtained. The participants selected into our research were from a single-center of adult MMD patients between August 2012 and January 2018. The inclusion criteria were following: 1) patients diagnosed with MMD according to guidelines published by the Research Committee on MMD ${ }^{16} ; 2$ ) patients treated by encephalo-duro-arterio-synangiosis (EDAS) alone; 3 ) patients performed with preoperative and postoperative cerebral angiography after revascularization; 4) patients older than $18 \mathrm{yrs}$. Patients with age less than 18 years old, moyamoya syndrome (MMS), other bypass procedures, posterior cerebral artery (PCA) involvement and unwilling to participate in the research were excluded. Finally, a total of 86 patients (97 hemispheres) were recruited into the research. Clinical data on gender, age, onset presentation, past medical history, modified Rankin Scale (mRS) on admission, and radiological findings were collected.

\section{Evaluation of collateral vasculature}

Preoperative collateral vasculature was evaluated by two independent neurosurgeons who were blinded to surgical information based on the criteria proposed by Gerasimos Baltsavias et al. ${ }^{17}$. The collateral circulation system was defined as follows: (1) intracerebral anastomosis, including the direct connections of striate arteries with the medullary arteries of the hemispheres; (2) durocortical anastomosis, consisting of collaterals between major intracranial and extracranial arteries 
(including ophthalmic artery (OphA), middle meningeal artery (MMA), superficial temporal artery (STA), and occipital artery (OcciA)); (3) leptomeningeal anastomosis, consisting of the anastomoses of the PCAACA branches and PCA-MCA branches at the watershed zones. This system also included a leptomeningeal network fed by the uncal artery, a branch of the proximal anterior choroidal artery (AchA) or distal ICA (Figure 1).

\section{Surgical Treatment}

Patients who met the following criteria were considered as unsuitable for direct revascularization: 1 ) those with no available recipient vessel for direct anastomosis during operation; 2) those who experienced frequent transient ischemic attacks (TIAs) during preoperative period (more than 3 times within 3 months before surgery) and/or those who experienced recent cerebral infarction (within 3 months before surgery). EDAS is the preferred indirect bypass revascularization procedure in our institution, with branch of the superficial temporal artery (STA) as the main donor artery.

\section{Evaluation of postoperative neovascularization}

Postoperative collateral grading was evaluated by reviewing the lateral view of the external carotid artery (ECA) based on cerebral angiography at 6 months after surgery. Collateral evaluations were performed by two independent neurosurgeons who were not involved in the surgery and were blinded to the clinical characteristics of MMD patients. The newly developed collateral circulation was evaluated by using the Matsushima grading ${ }^{5,10}$. Matsushima grading system was defined as follows: grade 0 , no collaterals present in the target revascularization area; grade 1, collaterals neovascularization presented in less than 1/3 MCA territory; grade 2, collaterals presented between 1/3 and 2/3 MCA territory; grade 3 , collaterals presented in more than 2/3 territory of the MCA. We artificially determined levels 2 and 3 as "good" collateral formation, whereas levels 0 and 1 were determined as "poor" collateral formation (Figure 2).

\section{Clinical follow-up}

Score of mRS was used to evaluate neurological status on admission and at final follow-up conducted by clinic visit or telephone up to minimal 6 months after surgery. Functional improvement and deterioration were defined as decrease or increase of $\mathrm{mRS}$ scores, respectively. During the clinic follow-up, ischemic events, hemorrhage, epilepsy and death were included.

\section{Statistical Analysis}

Statistical analysis was performed with the use of SPSS (version 22.0., IBM Corp.). Continuous variables with normal distribution were compared using a t-test, and skew distribution using with Mann-Whitney test. Categorical variables were compared with the Pearson chi-square test or Fisher's exact test as appropriate. Multivariate logistic regression analysis was conducted, including factors with $\mathrm{P}<0.05$ in the univariate analysis and factors reported in previous literature or according to clinical experience. Odds 
ratios (ORs) and 95\% confidence intervals (Cls) for good neovascularization were calculated. A p value < 0.05 was set as statistically significant.

\section{Results}

\section{Patient Demographics and Clinical Presentation}

Of 86 adult MMD patients (97 hemispheres) treated by EDAS, 49 females and 37 males (female/male ratio=1.32:1) were included, with $34.06 \pm 13.03$ years (range18-56 years) in average age. Tha number (percentage) of MMD patients with ischemic manifestation, hemorrhagic symptom and headache were 45 (52.3\%), $32(37.2 \%)$ and 9 (10.4\%), respectively. Nine (10.5\%) cases had a history of prior contralateral indirect bypass revascularization. Preoperative collateral characteristic included intracerebral anastomosis in 49 (50.5\%) hemispheres, duro-cortical anastomosis in 19 (19.6\%) hemispheres, and leptomeningeal anastomosis in 29 (29.9\%) hemispheres, respectively. (Table 1)

\section{Table 1. Clinical characteristics of adult patients with MMD.}

\begin{tabular}{ll} 
Variables & No of patients (n, \%) \\
\hline No. of patients (hemispheres) & $86(97)$ \\
\hline Age (yrs) & $34.06 \pm 13.03$ \\
\hline Age classification & \\
\hline$\leq 25$ & $25(29.1 \%)$ \\
\hline$>25$ & $61(70.9 \%)$ \\
\hline Genger (males) & $37(43.0 \%)$ \\
\hline Onset type & \\
\hline Hemorrhagic & $45(52.3 \%)$ \\
\hline Ischemic & $32(37.2 \%)$ \\
\hline Headache & $9(10.4 \%)$ \\
\hline Previous indirect revascularization & $9(10.5 \%)$ \\
\hline Collateral characteristic & \\
\hline Intracerebral anastomosis & $49(50.5 \%)$ \\
\hline Duro-cortical anastomosis & $19(19.6 \%)$ \\
\hline Leptomeningeal anastomosis & $29(29.9 \%)$ \\
\hline DSA follow-up (mos) & $9.15 \pm 5.47$
\end{tabular}




\section{Potential Risk Factors for Neovascularization After Indirect Bypass}

Of the 97 hemispheres treated by indirect bypass, 56 (57.7\%) cases had good postoperative neovascularization and $41(42.3 \%)$ had poor. There was no significant difference in DSA follow-up time between groups. Univariate analysis showed that younger age was significantly associated with good neovascularization $(P<0.001)$, whereas hemorrhagic onset and smoking were associated with poor neovascularization $(\mathrm{P}<0.05)$ (Table 2). Out of 29 hemispheres with leptomeningeal anastomosis, 24 (58.5\%) cases had poor neovascularization compared to 5 (8.9\%) cases with intracerebral anastomosis had good neovascularization $(\mathrm{P}<0.001)$. Consistently, good and poor neovascularization was observed in $66.1 \%$ and $29.3 \%$ hemispheres with intracerebral anastomosis, respectively $(P<0.001)$. The preoperative duro-cortical anastomotic had no association with postoperative neovascularization $(P>0.05)$. Multivariate analysis was conducted with the aforementioned significant factors. Age $(P<0.001$, OR [95\%Cl], 0.827 [0.793-0.916]), clinical onset type $(P<0.001,0 R[95 \% \mathrm{Cl}], 0.138$ [0.054-0.353]) and collateral anastomosis ( $P<0.001,0 R$ [95\% Cl], 2.984 [2.031-5.437]) were identified as independent predictors for neovascularization after indirect bypass surgery (Table 2).

Table 2. Logistic regression analysis of predictors for postoperative collateral formation 
Variables

Neovascularization $\quad P$ value

Good Poor Univariate Multivariate OR $(95 \% \mathrm{Cl})$

$(n=56) \quad(n=41)$

Age, yrs

Mean

$19.49 \pm$

$29.20 \pm$

$<0.001^{*}$

$<0.001^{*}$

0.827

13.74

15.41

$(0.793-$

$0.916)$

$\begin{array}{llll}18-25 & 34(60.7 \%) & 9(21.9 \%) & <0.001 \text { * } \\ >25 & 22(39.3 \%) & 32(78.1 \%) & \end{array}$

Male sex

$24(42.8 \%) \quad 18(43.9 \%) \quad$ NS

Onset type

Hemorrhagic

$4(7.1 \%)$

$17(41.5 \%) \quad<0.001 * \quad<0.001$

0.138

$(0.054-$

$0.353)$

Ischemic

$52(92.9 \%) \quad 24(58.5 \%)$

Risk factors

Hypertension

$7(12.5 \%) \quad 5(12.2 \%) \quad$ NS

Diabetes

$3(5.4 \%) \quad 0(0 \%) \quad$ NS

Alcohol abuse

$1(1.8 \%)$

$1(2.4 \%)$

NS

Smoking

$2(3.6 \%)$

$4(9.8 \%)$

NS

Hyperlipidemia

$2(3.6 \%)$

2(4.9\%)

NS

DSA findings

Unilateral MMD

$2(3.6 \%)$

$4(9.8 \%)$

NS

Suzuki stage

NS

\begin{tabular}{lcll}
$\square$ & $0(0.0 \%)$ & $1(1.7 \%)$ & NS \\
$\square$ & $2(3.6 \%)$ & $5(12.2 \%)$ & NS \\
$\square$ & $18(32.1 \%)$ & $13(31.7 \%)$ & NS \\
\hline & $24(42.9 \%)$ & $10(24.4 \%)$ & NS \\
$\square$ & $9(16.1 \%)$ & $10(24.4 \%)$ & NS \\
$\square$ & $3(5.4 \%)$ & $2(4.8 \%)$ & NS
\end{tabular}


Collateral anastomosis

$\begin{array}{lll}<0.001^{*} \quad<0.001^{*} \quad & 2.984 \\ & & (2.031- \\ & 5.437)\end{array}$

\begin{tabular}{lccl}
\hline Leptomeningeal & $5(8.9 \%)$ & $24(58.5 \%)$ & $<0.001 *$ \\
\hline Intracerebral & 37 & $12(29.3 \%)$ & $<0.001 *$ \\
& $(66.1 \%)$ & & \\
\hline Duro-cortical & $14(25 \%)$ & $5(12.2 \%)$ & NS \\
& & & \\
\hline Mean DSA follow-up, mos & $9.06 \pm$ & $8.15 \pm$ & NS \\
& 5.35 & 6.15 &
\end{tabular}

NS $=$ not significant $(P>0.05)$. Values are a number of hemispheres $(\%)$ unless otherwise indicated. Mean values are presented \pm SD.* $P<0.05$

\section{Long-Term Outcome}

During the average follow-up of $19.34 \pm 4.89$ months, hemispheres with poor neovascularization had no significant difference in the incidence of recurrent ischemia, rehemorrhage and seizure attack compared to those with good neovascularization in the entire series $(P>0.05)$. Follow-up mRS in $0-2$ was more commonly observed in hemispheres with good neovascularization ( $98.2 \%$ vs $85.4 \%, \mathrm{P}=0.039)$. Hemispheres in good neovascularization had lower follow-up mRS score than those in the poor neovascularization subgroup ( $0.60 \pm 0.70$ vs $1.06 \pm 1.13, \mathrm{P}=0.002)$. Hemispheres in the good neovascularization had better long-term improvement and deterioration outcome than those in the poor neovascularization (improvement: $94.6 \%$ vs $80.5 \%, P=0.014$; deterioration: $1.8 \%$ vs $12.2 \%, P=0.036$ ) (Table 3).

\section{Table 3. Long-term outcomes of patients}


Neovascularization

\begin{tabular}{llll}
\hline Variables & Good $(\mathrm{n}=56)$ & Poor $(\mathrm{n}=41)$ & P value \\
\hline Mean follow-up time, mos & $20.0 \pm 12.3$ & $20.9 \pm 12.6$ & 0.575 \\
\hline Recurrent symptoms & & & \\
\hline Cerebral hemorrhage & $1(1.8 \%)$ & $2(4.9 \%)$ & $\mathrm{NS}$ \\
\hline Cerebral ischemia & $8(14.4 \%)$ & $4(9.8 \%)$ & $\mathrm{NS}$ \\
\hline Seizure & $1(1.8 \%)$ & $1(2.4 \%)$ & $\mathrm{NS}$ \\
\hline Follow-up mRS & $0.60 \pm 0.70$ & $1.06 \pm 1.13$ & $0.002^{\star}$ \\
\hline $0-2$ & $55(98.2 \%)$ & $35(85.4 \%)$ & $0.039^{\star}$ \\
\hline$>3$ & $1(1.8 \%)$ & $6(14.6 \%)$ & \\
\hline Long-term outcome & & & \\
\hline Improvement & $53(94.6 \%)$ & $33(80.5 \%)$ & $0.049^{*}$ \\
\hline Unchanged & $2(3.7 \%)$ & $3(7.3 \%)$ & NS \\
\hline Deterioration & $1(1.8 \%)$ & $35(12.2 \%)$ & $0.036^{*}$ \\
\hline Death & $0(0.0 \%)$ & $0(0.0 \%)$ & NS
\end{tabular}

Good and poor refer to good neovascularization and poor neovascularization, respectively. Values are a number of hemispheres (\%) unless otherwise indicated. Mean values are presented \pm SD. ${ }^{*} p<0.05$.

\section{Discussion}

The effect of indirect revascularization on MMD relied on the neoangiogenesis from the vascularized donator into the cortical brain, which might act the same role in prefusion improvement and prevention in recurrence stroke as direct or combined bypass ${ }^{18,19}$. Previous reports investigated that effect in neovascularization was associated with favorable long-term outcome for patients with MMD, and potential predictors for postoperative neovascularization was of great value to provide standard for elective surgical procedure before surgery ${ }^{20}$. Thus far, few studies had reported potential risk factors for neovascularization after indirect bypass. In this research, we attempted to investigate the relationship between postoperative neovascularization and a variety of possible factors.

Our findings showed that good neovascularization was achieved in 56 (57.7\%) hemispheres after indirect bypass. The presence of preoperative collateral circulation system acted as independent risk factor for postsurgical neovascularization $(P<0.001,0 R$ [95\% Cl], 2.984 [2.031-5.437]). Preoperative intracerebral anastomosis predicted good neovascularization after surgery, whereas leptomeningeal anastomosis was a predictor of poor neovascularization. As it is known that cerebral ischemia indicated a potential signal 
for triggering moyamoya vessels and collateral growth in the progress of $M M^{21}$. As intracerebral anastomosis directly connects striate arteries with the medullary arteries, the main source of blood perfusion in cerebral cortex was leptomeningeal collateral, such as the anastomosis of the PCA-ACA branches and PCA-MCA branches at the watershed zones. Piao reported the hemisphere with extensive basal moyamoya vessels exhibited impaired perfusion and metabolism in cerebral cortex ${ }^{22}$. Takahashi et al. also reported that severe ischemia was essential for the formation of new vessels ${ }^{23}$. The transdural anastomosis developed in the advanced Suzuki stage, such as meningeal middle artery, ethmoid moyamoya and vault moyamoya vessels ${ }^{24-26}$. Thus, we hypothesized that hemisphere with intracerebral anastomosis was more likely to suffer cerebral ischemia than those with leptomeningeal collateral, stimulating the development of collateral development after surgical revascularization. Our study was a practical interpretation of association between preoperative collateral anastomosis and postoperative neovascularization, and the mechanisms behind this phenomenon remain to be clarified by future studies.

Previous studies demonstrated that indirect bypass was more effective for patients with young age and ischemic onset symptom 4,27 . In the study, 34 (60.7\%) cases younger than 25 years had good neovascularization compared to 22 (39.3\%) cases older than 25 years $(P<0.001,0 R$ [95\% Cl], 0.827 [0.793-0.916]), indicating age might be a relatively significant predictor. Previous literature reported that hemisphere in young age was associated with better cerebrovascular reactivity and higher secretion of angiogenic peptides, vascular endothelial growth factor (VEGF) stimulating collateral growth than in old age when suffering from cerebral ischemia ${ }^{28-30}$. However, Zhao reported that age was not an independent predictor for neovascularization after indirect bypass in the entire series, but the predictor of MMD patients in the hemorrhagic-onset type, which differed from our results ${ }^{15}$. As for age was not the most influential predictor affecting surgical effect, it still needed to explore further.

In addition, in our series, $41.5 \%$ hemispheres in the hemorrhagic had poorer neovascularization than $7.1 \%$ in the ischemic $(\mathrm{P}<0.001, \mathrm{OR}[95 \% \mathrm{Cl}], 0.138$ [0.054-0.353]). Previous reports demonstrated that hemorrhagic onset type was a potential predictor for neovascularization after indirect bypass ${ }^{15,31}$. As is known the hemispheres with normal perfusion in the hemorrhagic were more common than the ischemic group. The MMD patients with hemorrhage tended to be diagnosed in early stage of the disease due to abrupt presence of brain hemorrhage, whereas the ischemic tended to be in the advanced stage ${ }^{32}$. We hypothesized that cerebral revascularization itself might be a destruction to the vulnerable blood supply from extracranial arteries for hemorrhagic MMD patient receiving surgery treatment too early, which could cause an adverse effect on postoperative neovascularization. Hence, the general superiority of direct bypass over indirect bypass for MMD patients should not be ignored, especially for the MMD patients with hemorrhage.

According to previous reports, good neovascularization was associated with better long-term outcomes ${ }^{33}$, ${ }^{34}$. Better neurological status and more improved outcomes were observed in hemispheres with good neovascularization, suggesting the effect of revascularization was crucial to the long-term outcome for 
adult MMD patients (improvement: $94.6 \%$ vs $80.5 \%, \mathrm{P}=0.014$; deterioration: $1.8 \%$ vs $12.2 \%, \mathrm{P}=0.036$ ). However, there was no significant difference in recurrent ischemia, rehemorrhage and seizure between hemispheres with poor and good neovascularization $(P>0.05)$. This finding was different from results reported by Arias et $\mathrm{al}^{35}$, due to the comparatively larger sample size of the study

\section{Limitations}

The current study had a few limitations as follow. First, our study was a retrospective research in a single center, and selection bias might exist. The conclusion needed a multicenter research for further verification. Second, sample size of the study was small, which need more patients enrolled to confirm the conclusion. Third, the current study did not elucidate the potential pathophysiology, and future studies were needed to further address this issue. Fourth, the main indirect bypass included in the study is EDAS, so there was no basis for the evaluation of the other indirect bypass surgery. Fifth, the study lacked data on cerebral perfusion, and radiological brain perfusion examination would be the next.

\section{Conclusions}

Hemorrhagic onset and old age predict poor neovascularization after indirect bypass, while duro-cortical anastomosis and intracerebral anastomosis predict good neovascularization. Good neovascularization was associated with better long-term outcomes. The current study provides a basis for the selection of surgical procedure for MMD candidates.

\section{Declarations}

\section{Acknowledgments}

Not applicable.

\section{Authors' contributions}

Kefang Dai. and Zhiyong Shi wrote the main manuscript text, We want them both to be common first authors.and Xingju Liu. prepared figures 1-2.Rong Wang and Yan Zhang prepared tables.Dong Zhang is responsible for the whole article.All authors reviewed the manuscript.

\section{Funding}

The authors declare that they have no sources of funding related to the manuscript

\section{Availability of data and materials}

All data generated or analyzed during this study are included in this published article 
Ethics approval and consent to participate

This study was approved by the institutional review board of Beijing Tiantan Hospital, Capital Medical University. We followed the guidelines outlined in the Declaration of Helsinki, Written informed consent was obtained from all patients.

\section{Consent for publication}

Not applicable

\section{Competing interests}

None of the authors have any conficts of interest to disclose.

\section{Author details}

Kefang Daia,b and Zhiyong Shi c, Xingju Liu a ,Rong Wang a, Yan Zhanga Dong Zhanga,*

aDepartment of Neurosurgery, Beijing Tiantan Hospital,Capital Medical University, China National Clinical Research Center for Neurological Diseases, Beijing, China

bXingtai Third Hospital,Xingtai, Hebei,China.

c Nanjing drum tower hospital $\nabla$ the affiliated hospital of Nanjing university medical school $\nabla N a n j i n g \rrbracket$ Jiangsu province $₫$ China $₫ 210007$

* Corresponding author at: Department of Neurosurgery, Beijing TiantanHospital, Capital Medical University, No.119 South Fourth Ring West Road, Fengtai District, Beijing 00050, China. Fax: 8610 67096523. E-mail: zhangdong0660@aliyun.com

Kefang Dai, E-mail: hedou-1@163.com M.D

Zhiyong Shi, E-mail: szy1195156829@aliyun.com M.D

Xingju Liu, E-mail: liuxingju006@163.com M.D

Rong Wang, E-mail: ronger090614@126.com M.D., Ph.D.

Yan Zhang, E-mail: yanzhang135@163.com M.D., Ph.D.

Dong Zhang, E-mail: zhangdong0660@aliyun.com M.D., Ph.D.

\section{References}


1. Han $\mathrm{DH}, \mathrm{Nam} \mathrm{DH}, \mathrm{Oh} \mathrm{CW}$. Moyamoya disease in adults: characteristics of clinical presentation and outcome after encephalo-duro-arterio-synangiosis. Clin Neurol Neurosurg 1997;99 Suppl 2:S151-155.

2. Hoshino H, Izawa Y, Suzuki N, Research Committee on Moyamoya D. Epidemiological features of moyamoya disease in Japan. Neurol Med Chir (Tokyo) 2012;52:295-298.

3. Kuriyama S, Kusaka Y, Fujimura M, et al. Prevalence and clinicoepidemiological features of moyamoya disease in Japan: findings from a nationwide epidemiological survey. Stroke 2008;39:4247.

4. Goda $\mathrm{M}$, Isono $\mathrm{M}$, Ishii $\mathrm{K}$, Kamida $\mathrm{T}$, Abe T, Kobayashi $\mathrm{H}$. Long-term effects of indirect bypass surgery on collateral vessel formation in pediatric moyamoya disease. J Neurosurg 2004;100:156-162.

5. Nakashima H, Meguro T, Kawada S, Hirotsune N, Ohmoto T. Long-term results of surgically treated moyamoya disease. Clin Neurol Neurosurg 1997;99 Suppl 2:S156-161.

6. Scott RM, Smith JL, Robertson RL, Madsen JR, Soriano SG, Rockoff MA. Long-term outcome in children with moyamoya syndrome after cranial revascularization by pial synangiosis. J Neurosurg 2004;100:142-149.

7. Kim DS, Huh PW, Kim HS, et al. Surgical treatment of moyamoya disease in adults: combined direct and indirect vs. indirect bypass surgery. Neurol Med Chir (Tokyo) 2012;52:333-338.

8. Kuroda S, Houkin K. Moyamoya disease: current concepts and future perspectives. Lancet Neurol 2008;7:1056-1066.

9. Macyszyn L, Attiah M, Ma TS, et al. Direct versus indirect revascularization procedures for moyamoya disease: a comparative effectiveness study. J Neurosurg 2017;126:1523-1529.

10. Matsushima T, Inoue T, Suzuki So, Fujii K, Fukui M, Hasuo K. Surgical treatment of moyamoya disease in pediatric patients-comparison between the results of indirect and direct revascularization procedures. Neurosurgery 1992;31:401-405.

11. Mizoi K, Kayama T, Yoshimoto T, Nagamine Y. Indirect revascularization for moyamoya disease: is there a beneficial effect for adult patients? Surg Neurol 1996;45:541-548; discussion 548-549.

12. Morshed RA, Abla AA, Murph D, et al. Clinical outcomes after revascularization for pediatric moyamoya disease and syndrome: A single-center series. J Clin Neurosci 2020;79:137-143.

13. Starke RM, Komotar RJ, Hickman ZL, et al. Clinical features, surgical treatment, and long-term outcome in adult patients with moyamoya disease. Clinical article. J Neurosurg 2009;111:936-942.

14. Deng X, Gao F, Zhang D, et al. Effects of different surgical modalities on the clinical outcome of patients with moyamoya disease: a prospective cohort study. J Neurosurg 2018;128:1327-1337.

15. Zhao Y, Li J, Lu J, et al. Predictors of neoangiogenesis after indirect revascularization in moyamoya disease: a multicenter retrospective study. J Neurosurg 2019:1-11.

16. Fukui M. Guidelines for the diagnosis and treatment of spontaneous occlusion of the circle of Willis ('moyamoya' disease). Research Committee on Spontaneous Occlusion of the Circle of Willis (Moyamoya Disease) of the Ministry of Health and Welfare, Japan. Clin Neurol Neurosurg 1997;99 Suppl 2:S238-240. 
17. Baltsavias $\mathrm{G}$, Khan N, Valavanis A. The collateral circulation in pediatric moyamoya disease. Childs Nerv Syst 2015;31:389-398.

18. Adelson PD, Scott RM. Pial synangiosis for moyamoya syndrome in children. Pediatr Neurosurg 1995;23:26-33.

19. Acker G, Fekonja L, Vajkoczy P. Surgical Management of Moyamoya Disease. Stroke 2018;49:476482.

20. Kim T, Oh CW, Bang JS, Kim JE, Cho WS. Moyamoya Disease: Treatment and Outcomes. J Stroke 2016;18:21-30.

21. Coyle P, Heistad DD. Development of collaterals in the cerebral circulation. Blood Vessels 1991;28:183-189.

22. Piao R, Oku N, Kitagawa K, et al. Cerebral hemodynamics and metabolism in adult moyamoya disease: comparison of angiographic collateral circulation. Ann Nucl Med 2004;18:115-121.

23. Takahashi A, Sawamura Y, Houkin $K$, Kamiyama $H$, Abe $H$. The cerebrospinal fluid in patients with moyamoya disease (spontaneous occlusion of the circle of Willis) contains high level of basic fibroblast growth factor. Neurosci Lett 1993;160:214-216.

24. Yamada I, Himeno Y, Suzuki S, Matsushima Y. Posterior circulation in moyamoya disease: angiographic study. Radiology 1995;197:239-246.

25. Suzuki J, Kodama N. Moyamoya disease-a review. Stroke 1983;14:104-109.

26. Storey A, Michael Scott R, Robertson R, Smith E. Preoperative transdural collateral vessels in moyamoya as radiographic biomarkers of disease. J Neurosurg Pediatr 2017;19:289-295.

27. Imai H, Miyawaki S, Ono H, Nakatomi H, Yoshimoto Y, Saito N. The importance of encephalo-myosynangiosis in surgical revascularization strategies for moyamoya disease in children and adults. World Neurosurg 2015;83:691-699.

28. Croley AN, Zwetsloot KA, Westerkamp LM, et al. Lower capillarization, VEGF protein, and VEGF mRNA response to acute exercise in the vastus lateralis muscle of aged vs. young women. J Appl Physiol (1985) 2005;99:1872-1879.

29. Shweiki D, Itin A, Soffer D, Keshet E. Vascular endothelial growth factor induced by hypoxia may mediate hypoxia-initiated angiogenesis. Nature 1992;359:843-845.

30. Wei L, Erinjeri JP, Rovainen CM, Woolsey TA. Collateral growth and angiogenesis around cortical stroke. Stroke 2001;32:2179-2184.

31. Zhao M, Zhang D, Wang S, Zhang Y, Deng X, Zhao J. The Collateral Circulation in Moyamoya Disease: A Single-Center Experience in 140 Pediatric Patients. Pediatr Neurol 2017;77:78-83.

32. Yin H, Liu X, Zhang D, et al. A Novel Staging System to Evaluate Cerebral Hypoperfusion in Patients With Moyamoya Disease. Stroke 2018;49:2837-2843.

33. Yoshida Y, Yoshimoto T, Shirane R, Sakurai Y. Clinical course, surgical management, and long-term outcome of moyamoya patients with rebleeding after an episode of intracerebral hemorrhage: An extensive follow-Up study. Stroke 1999;30:2272-2276. 
34. Zhao Q-S, Wang G, Xiao H-J, et al. [Characteristics of collateral circulation in adult moyamoya disease based on modified Suzuki staging]. Nan Fang Yi Ke Da Xue Xue Bao 2018;38:483-489.

35. Arias EJ, Dunn GP, Washington CW, et al. Surgical Revascularization in North American Adults with Moyamoya Phenomenon: Long-Term Angiographic Follow-up. J Stroke Cerebrovasc Dis 2015;24:1597-1608.

\section{Figures}
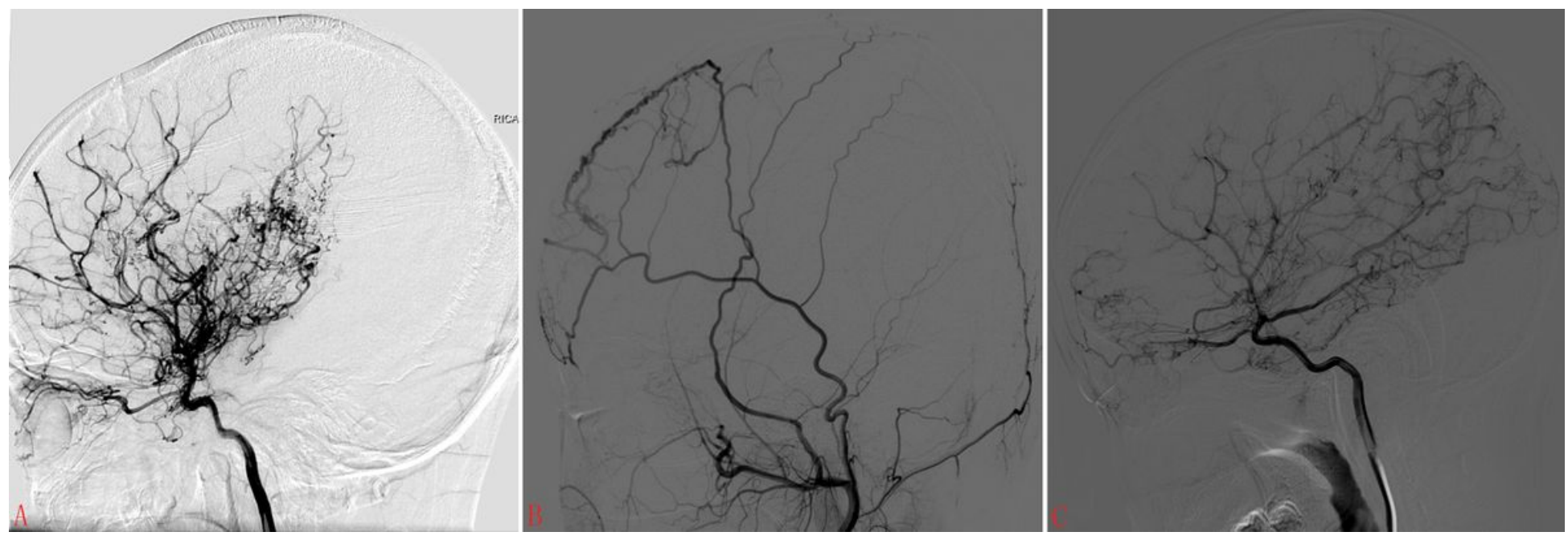

Figure 1

Evaluation of collateral vasculature. A. Intracerebral anastomosis, including the direct connections of striate arteries with the medullary arteries of the hemispheres. B. Duro-cortical anastomosis, including middle meningeal artery (MMA) to cerebrum. C. Leptomeningeal anastomosis, consisting of PCA-MCA branches network at the watershed zones. 

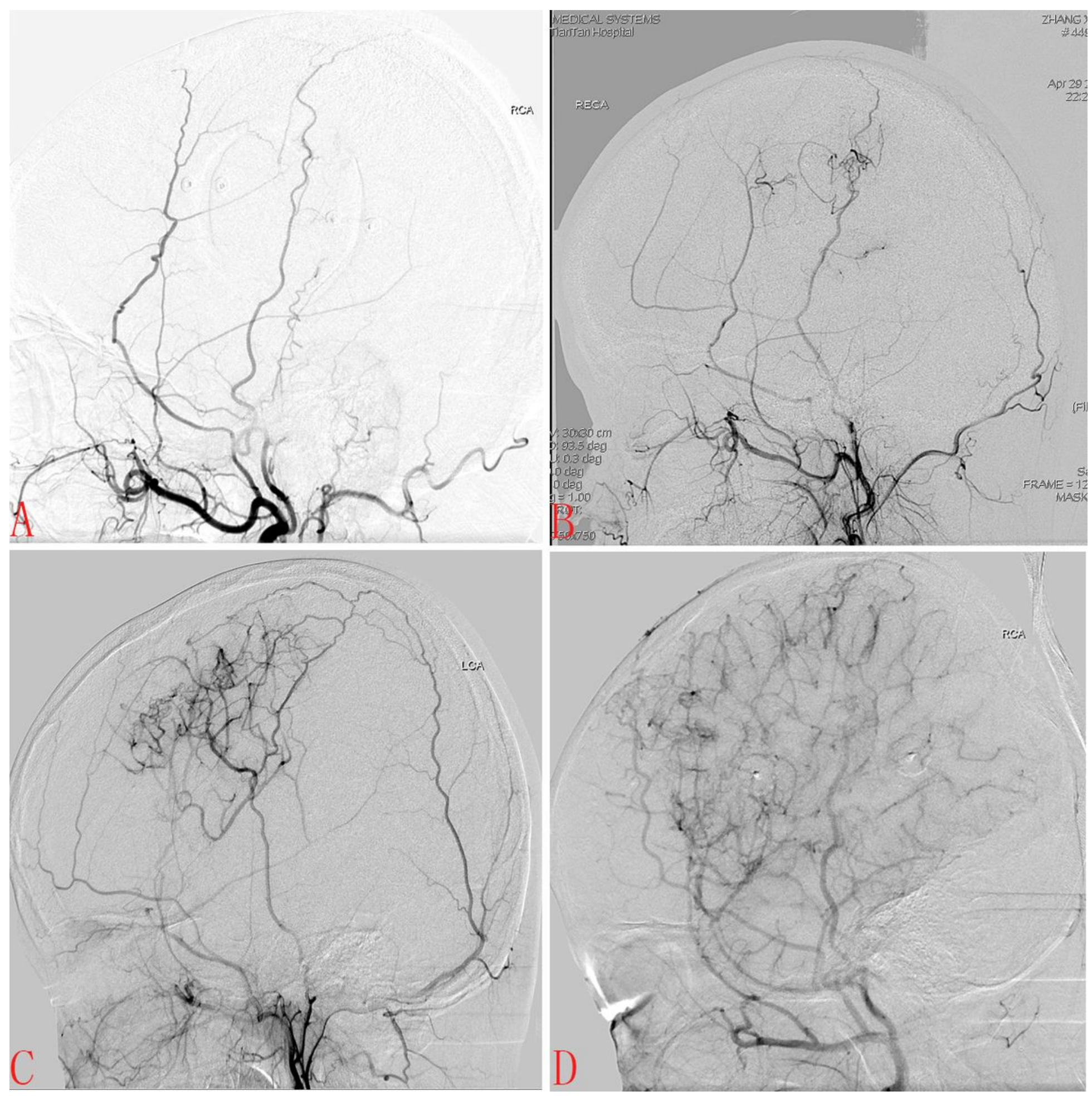

Figure 2

Evaluation of postsurgical neovascularization based on Matsushima gradings. A. Level 0 (null): no obvious neovascularization. B. Level 1 (localized): neovascularization covering less than 1/3 MCA territory. C. Level 2 (moderate): neovascularization covering between 1/3 and 2/3 MCA territory. D. Level 3 (abundant): new developed collaterals covering more than 2/3 MCA territory. 